$\xi=-1$

\title{
Implementation of the centralized control system for drone training
}

\author{
Bong-Hyun Kim * \\ Department of IT Convergence, U1 University, 310 Daehak-ro, Yeongdong-eup, Yeongdong-gun, Chungcheongbuk-do, 29131, Republic \\ of Korea \\ *Corresponding authorE-mail: bhkim@ul.ac.kr
}

\begin{abstract}
Background/Objectives: The drones became a representative item in the IoT era. However, there is no drone pilot test system that can safely train this in the education field. Drones have very dangerous structural problems, so it is very necessary to practice them easily. Therefore, it is necessary to develop a system that can control the drones safely and easily while controlling them.

Methods/Statistical analysis: In this paper, we will develop software for controlling a dedicated board platform that can securely perform ground testing by mounting four drones of motor and drive on a board (PCB). To this end, we supported various control IMU (Inertial Measurement Unit) boards for attitude control by using sensor which is the core technology of drone flight control. Also, Acceleration Data, Angular Velocity Data, Earth Magnetic Field Data, and Atmospheric Pressure Data for maintaining the altitude were used for the drone flight.

Findings: In the implemented central control system, the AT chip is built in and designed to perform all control related to the flight of the drone. In addition, since it is an embedded system, we have programmed the attitude control using the sensor, the motor output setting, and the controller connection information. The CPU required for drones control can be replaced with various types of controllers besides Fno Arduino, UNO, Muiltiwii. For this purpose, the main PCB is designed so that the power supply terminal can be used for each CPU. Finally, it was developed as a setup program to correct the sensor and output of the drone.

Improvements/Applications: The system implemented in this paper can easily control the drone. In addition, acceleration, angular velocity, geomagnetic field, air pressure sensor, GPS, etc. necessary for drone control can be utilized by stabilizing the initial set value. In other words, the zero point of the sensor can be captured and the signal appropriate to the current state of the drone can be stored in the processor.
\end{abstract}

Keywords: Drone Test Software; Multiwii, Drone Training; Arduino; Flight Control Board; Signal Process

\section{Introduction}

The drones became a representative item of the IoT era. Drones are "unmanned flying devices" that fly in the automatic and semiautomatic manner by a remote control or preprogrammed route from the ground without pilots flying, or autonomous flight based on artificial intelligence. The industrial importance of drones and the market size are increasing day by day. Drones' aviation, communication, sensor, and software technologies have a significant impact on related fields. It is also an optimal test bed for applying and verifying common core technologies of the fourth industrial revolution such as artificial intelligence, Internet of objects and sensors. However, there is no drone pilot test and practice system that can safely train it in the education field. Currently, some DIY drones are conducting test exercises against a small number of personnel, but it is urgent to prepare measures for safety accidents. Drones have very dangerous structural problems, so it is essential to have an environment where you can practice easily. In fact, even though skilled people only have to fly in confined spaces, those who are new to drones can easily assemble drones. However, this causes many people to be injured and leading to major personal accidents [1].

Generally, electric motors of home electric fans are usually 2,000 $\sim$ 3,000 RPM. The motors used in general drones are usually be- tween 40,000 and 50,000 RPM. The blade, called a blade, has a sharp end. It is very dangerous for an educational institution to assemble and test drones that have four high-speed rotating wings without any safety training equipment. In the case of Figure 1 shows the accident caused by the drones. Drones are the first and most dangerous and careful process of setting the motor. Most injured areas are weak wrists, face, and legs. Among the most injured areas, the inner wrist is more dangerous as it can be severed from the artery.
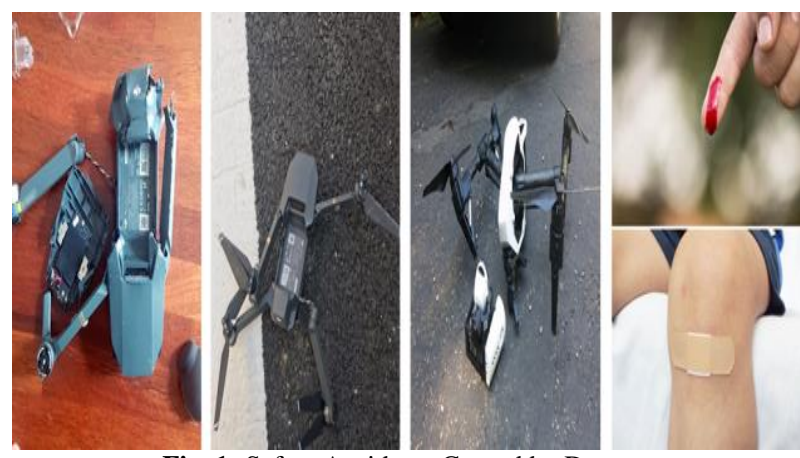

Fig. 1: Safety Accidents Caused by Drones. 
However, it is a very useful system if you fly the drones after having drone training to prevent safety accidents. Especially, the nextgeneration flying drones are a very flexible base for new technologies to emerge and are expected to have a major impact on human society, along with cameras, lighting, and sensors. Currently, hobby and industrial drones that can be easily accessed from around are mostly multicopter type. The existing hobby radiocontrolled aircraft is difficult to maneuver, and it is difficult to secure visibility and landing. The multi-copter drones are easy to land and fly. Especially, recently, it is possible to use the developed GPS to control the vehicle over long distances of several $\mathrm{Km}$ or more outside the watch range of the operator. In addition, obstacle avoidance function has been added to prevent many collisions and many useful functions have been developed [2] [3].

However, in terms of safety education and control, drone is difficult to transmit in basic position or altitude because there is no equipment such as transponder (position transmitter) mounted on aircraft. In addition, since the controller relies on GPS without a precise attitude meter, directional instrument, etc., and is dependent on a monitor through a camera, it is limited in precision flight. As a result, it increases the risk of collision with other airplanes, buildings, and natural objects, and safety accidents occur frequently.

Therefore, in this paper, we developed a configuration program to correct the sensor and output of the drone, and implemented the system to control the drone in the center. For this purpose, the acceleration, angular velocity, geomagnetic field, air pressure sensor, and GPS required to control the drone are designed to stabilize the initial set values. In addition, the zero point of the sensor is set so that the signal corresponding to the current state of the drone is stored in the processor. Finally, the implementation system is used to implement interoperability with the pilot test platform so that drone training and pilot practice tests can be safely performed.

\section{Drone characteristics and operating envi- ronment}

\subsection{Characteristics}

Drones are included in lightweight UAVs that are small in size, light in weight and developed in various forms. The drones do not need asphalt or concrete pavements like regular aircraft because of their short haul, landing and maneuverability. If you have about 20 hours of flying experience, you will get a full-length pilot's license after passing the qualification test, so you can fly and there are many people who run it for leisure. In recent years, the development of drones and the publicity of mass media tend to slow down the expansion of lightweight aircraft in Korea. However, unlike drones, people are able to board and move with cargo, the cost of maintenance is low, and freedom of activity is a big advantage because they receive relatively less control from the air traffic control agency.

The characteristics of the drone can be summarized in four major ways. First, it is possible to fly in place. In situ flying is the most advantageous feature of a flywheel because it maintains a constant altitude and direction while staying in the air. Second, side and back flight is possible. Fixed wings make the unimaginable lateral and rearward flight possible by tilting the rotation side laterally or backward. Third, vertical takeoff and landing is possible. The characteristic of being able to fly in place is that it is possible to take off and land at the place even if only the space of the allowable length of the rotor blade is secured. Fourth, it is dynamic instability. Dynamic instability refers to a state in which the amplitude is gradually increased without decreasing the vibration with time when the external force is applied to the object in equilibrium state. The flywheel has this characteristic when it flies, so if the pilot does not perform the corrective operation, it can go to a dangerous situation with an increase in the amplitude [4] [5].

\subsection{Operating environment}

The drones should be composed of the most basic and easy-tomaneuver design, Quadcopter(with 4 rotating blades), 6, 8, etc., with even number of rotating blades varying in size, usage and price. Therefore, it is easy to cancel the torque, and it is possible to stably operate and collapse in an emergency. Since each rotary blade is designed to rotate clockwise or counterclockwise, it must be mounted on the motor to maintain the proper rotation direction when drone assembly or maintenance. Be careful that drone beginners often ignore this principle and fall erroneously into assembling the rotor.

The drone must be precisely sized and positioned so that the center of gravity is always within the stable range. As the operation time of general aircraft increases, the fuel consumption and the lift characteristics increase. However, the drone does not have the effect of increasing the lift according to the flight time because the battery is used as the basic power, but it has a disadvantage due to the decrease of the battery voltage. The fuselage is arranged in an $\mathrm{X}$-shape, a cross-shape, and an $\mathrm{H}$-shape so that four propeller positions are symmetrically mounted with two each. The center should be considered as the basis of the center of gravity by considering the battery first, and consideration should be given to the space where other electronic equipment and accessories are to be mounted [6].

Recently, the drones are mounted on the front of the camera or the camera mount(Gimbal system) at the bottom of the fuselage is attached to the bottom of the fuselage. The prepared body must be suspended in the air to check the $\mathrm{X}, \mathrm{Y}$, and $\mathrm{Z}$ axes to ensure a stable center position and consider other accessories or equipment mounting. Of course, some weight imbalance can be solved using software [7] [8]. However, this is undesirable because it will give the propeller motor a load and affect safe landings during emergency procedures. Also, the landing gear is mounted on the bottom of the fuselage to maintain ground stability and to protect the fuselage.

Propellers are made of various materials such as titanium, carbon fiber, and plastic. They have different characteristics and angles, but the factors that influence their performance are size and twist angle. If each propeller is the same size, a propeller with a large twist angle is favorable for receiving lift, but because it needs to rotate faster, it is likely to become unstable due to the increased vibration. Depending on the type of drones, the small introductory hobby drone is equipped with a propeller protector, so it is safe to fly in the room or in a small space, and it can reduce damage when an external object touches it [9] [10]. Therefore, it is possible to reduce a lot of human injury that occurs in the present if the standard heavy drones are provided with the protector mounting.

The drone motor corresponds to an aircraft engine, and the multicopter motor has a revolution per minute of more than about 8,000 RPM. It controls the number of revolutions quickly and accurately according to the signal of the speed controller and enables stable flight. The motors are divided into compact and low cost brush motors with carbon brushes and brushless motors without brushes. Brush motors have a short life span, so it is difficult to show their ability when flying for about 10 hours. Brushless motors substitute their roles as electronic circuit elements instead of carbon brushes, enabling fast and precise motor control[11]. However, it is costly and vulnerable to heat, so if you continue to operate after a certain number of revolutions, efficiency will decrease.

An electric speed controller(ESC) connects the power between the battery and the motor. It adjusts the number of revolutions of each motor according to the signal of the flight regulator mounted on the fuselage, and adjusts the number of revolutions of the propeller. It functions to transmit the engine speed to the tire like a transmission of an automobile. However, the drones' transmissions perform more sophisticated and complex functions in that they control multiple motor rotations at the same time [12] [13].

Finally, the FCC is similar to the CPU of a computer, which corresponds to the drones' brains. It is a central processing unit that receives the signal sent from each sensor and radio control signal 
related to the movement of the drone, and sends it to the flight motor after processing. The circuit board incorporates various kinds of sensors and processing chips, and is designed in front of the body considering the center of gravity and the rapid change detection of each sensor.With the exception of the sensors, the most important part of the FCC is the multiple receivers that receive radio signals from the radio control. Each receiving device is connected to one of the wires and sent to the FCC to be used as a control signal for the drone flight.

\section{Design and implementation}

As drone technology and industry develop, systematic drone training and pilot training test are indispensable. The drone rotates the propeller at high speed to fly the drone structure in a manner that generates lift. Therefore, it is possible that a crash or secondary accident may occur during flight due to inadequate control, improper sensor calibration, error control of PID (Proportional Integral Differential) setting value of ESC (Electric Speed Controller).Therefore, in this paper, we developed a configuration program to correct the sensor and output of the drone, and implemented the system to control the drone in the center. For this purpose, the acceleration, angular velocity, geomagnetic field, air pressure sensor, and GPS required to control the drone are designed to stabilize the initial set values. In addition, the zero point of the sensor is set so that the signal corresponding to the current state of the drone is stored in the processor. Finally, the implementation system is used to implement interoperability with the pilot test platform so that drone training and pilot practice tests can be safely performed.

In this paper, we developed central control software using Multiwii Sauce Code. The Multiwii Sauce Code is the Programing Sauce Code of the Flight Control Board (FCB) using the ATmega series main chip. It provides various configuration to apply to various ATmega series FCB. Data transmission between PC and FCB via FCB digital / analog signal I / O control and USB type serial communication can be configured very easily. It contains various settings related to various types of drone so that various types of drone can be set. It supports various control IMU(Inertial Measurement Unit) board for posture control using sensor which is the core technology of drone flight control, so it is necessary to use Acceleration Data, Angular Velocity Data), Earth Magnetic Field Data (Magnetic Field Data), and atmospheric pressure data for maintaining altitude. The MultiWii sauce code is shown in figure 2 .
In the Config.h tab, you can select Drone Type, Main chip, IMU board and GPS board. You can set the output of Drone by adjusting throttle value. In the Def.h tab, detailed information and information according to each main chip are stored, so that the user can check the hardware connection information according to the Drone Main chip that the user wants to use. You can also change the port settings to match the drone you are using.

The Main Control Module contains an AT chip and is responsible for all control related to the flight of the drone. Since it is an embedded system, it uses the sensor to control attitude control, motor output setting, and controller connection information, and uploads it to the Main Control Module. ESC controls the motor by adjusting the voltage of the BLDC motor by the PWM (pulse width modulation) method through the control value of the main module. It also has a built-in voltage regulator to supply the rated $5 \mathrm{~V}$ voltage to the main board. The RF Control transceiver is capable of transmitting and receiving up to $2 \mathrm{~km}$ using the $2.4 \mathrm{GHz}$ bandwidth Ride frequency. There are 4 directional control buttons for Lift, Pitch, Roll, Yaw direction, and there are 3 step toggle switches for setting flight mode. As shown in figure 3 the MultiWii program display.

The final control system was developed as a configuration software to correct the sensor and output of the drone. Acceleration, angular velocity, geomagnetic field, air pressure sensor, GPS, etc. necessary to control the drone can be utilized by stabilizing the initial set value. In other words, the zero point of the sensor is captured and the signal corresponding to the current state of the Drone is stored in the processor.

Acceleration sensor value notation in central control software indicates ROLL, PITCH, $\mathrm{Z}$ value between 0 and 512 . The gyro sensor value indicates ROLL, PITCH, $\mathrm{Z}$ value between 0 and 512 . Also, the geomagnetic field is a MEMS gyro type, and values of ROLL, PITCH, YAW are indicated between 0 and 512. ANGLE stands for horizontal flight function and maintains a more stable posture. If it is not selected, it is set to ACRO (acrobatic) mode, which makes the drone very sensitive and difficult to maneuver. HORIZEN is designed to move like the ANGLE Mode if you manipulate the controls and the ACRO Mode if you manipulate them quickly.

The CPU required for drones control is configured to be able to replace various types of controllers in addition to Fno Arduino, UNO, MuiltiWii. For this purpose, the main PCB is designed so that the power supply terminal can be used for each CPU. //\#def ine GIMBAL

$/ /$ \#def ine BI

//\#def ine TRI

//\#def ine QUADP

\#̈def ine QUADX

//\#indef ine $V_{4}$

$/ /$ \#def ine $V_{6}$

//\#def ine HEXG

/ \#def ine HEX6X

//\#def ine HEX6H // New ModeI

THef ine HexhH

Het ine OCT0X8

Hdef ine OCTOFLATP

Andef ine OCTOFLATX

/ \#def ine FLVING_WING

//\#def ine VTAIL4

//\#def ine AIRPLANE

//\#def ine SINGLECOPTER

//\#def ine DUALCOPTER

//\#def ine HELI_120_CCPA

//\#def ine HELI_90_DEG

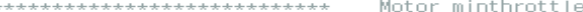

* Set the minimum throttle command sent to the ESC (Electronic Speed Controller)

This is the minimum value that allow motors to run at a idle speed $t /$

//\#def ine MINTHROTTLE $1300 / /$ for Turnigy Plush ESCs $10 \mathrm{~A}$

/\#def ine MINTHROTTLE $1120 / /$ for Super Simple ESCs 10A

/ \#def ine MINTHROTTLE $1064 / /$ special ESC (simonk)

//\#def ine MINTHROTTLE $1064 / /$ special ESC (simonk)

\#def ine MINTHROTTLE $1100 / /(*)$ (**)

* this is the maximum value for the ESCs at full power, this value can be increased up to $2000 *$ /

\#def ine MAXTHROTTLE 1950

Fig. 2: Multiwii Sauce Code. 


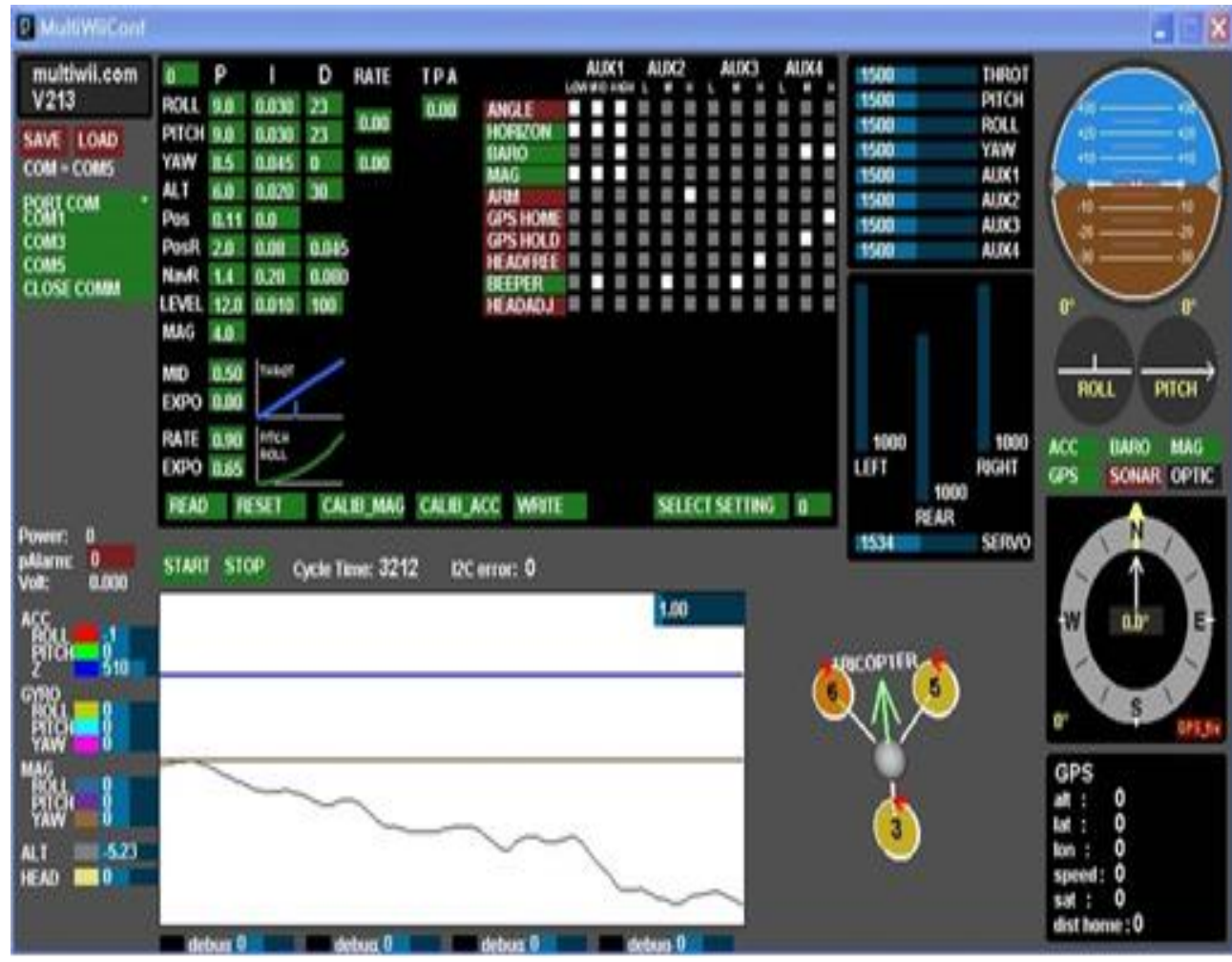

Fig. 3: Multiwii Control Display.

Testing the drones begins with a primary CPU selection. Therefore, this system is implemented so that various CPUs can be selected and used. In addition, we will test the ESC secondarily. In the case of a quad-copter, four ESCs and four motors are used, so the setting must be four times each. If you miss this process, the drones will not be able to fly normally. It is very important how accurate and convenient ESC can be set by default in the system. For this purpose, in the implementation system, the position of the interface connection terminal with the PC is designed so as not to interfere with the rotation of the propeller in order to facilitate the connection with the PC. As shown in table 1 the information for each mode in the MultiWii Control System.

Table 1: Multiwii Control System Mode Information

\begin{tabular}{|c|c|}
\hline Mode & Information \\
\hline Acceleration & $\begin{array}{l}\text { Acceleration sensor value is indicated as ROLL, } \\
\text { PITCH, Z value between } 0 \text { and } 512 \text {. }\end{array}$ \\
\hline Gyro & $\begin{array}{l}\text { Gyro sensor value is indicated as ROLL, PITCH, Z } \\
\text { value between } 0 \text { and } 512 \text {. }\end{array}$ \\
\hline $\begin{array}{l}\text { Geometry sys- } \\
\text { tem }\end{array}$ & $\begin{array}{l}\text { MEMS gyro type, the value of ROLL, PITCH, and } \\
\text { YAW should be between } 0 \text { and } 512 \text {. }\end{array}$ \\
\hline $\begin{array}{l}\text { Altitude \& Di- } \\
\text { rection }\end{array}$ & $\begin{array}{l}\text { ALT is the value of the air pressure sensor and its } \\
\text { value changes according to the pressure difference. It } \\
\text { is used to maintain altitude. The HEAD value indi- } \\
\text { cates the heading value of the gas. }\end{array}$ \\
\hline $\begin{array}{l}\text { Earth magnetism } \\
\text { Calibration }\end{array}$ & It catches the level of earth sensor. \\
\hline $\begin{array}{l}\text { Acceleration } \\
\text { Calibration }\end{array}$ & It catches the level of acceleration sensor. \\
\hline $\begin{array}{l}\text { Direction of } \\
\text { Drone \& Prop }\end{array}$ & $\begin{array}{l}\text { Displays the current direction of the drone in three } \\
\text { dimensions, and shows the pin information of each }\end{array}$ \\
\hline $\begin{array}{l}\text { No. } \\
\text { Level infor- } \\
\text { mation }\end{array}$ & $\begin{array}{l}\text { board connected to each propeller. } \\
\text { It shows Roll and Pitch value (slope) information } \\
\text { based on sensor value. }\end{array}$ \\
\hline Stick level & $\begin{array}{l}\text { It shows the output value according to manipulation of } \\
\text { the manipulator of the manipulator. }\end{array}$ \\
\hline PID setting & $\begin{array}{l}\text { This is the part that adjusts the speed and intensity of } \\
\text { the change of the voltage applied when controlling the } \\
\text { drone. The larger the value, the greater the movement } \\
\text { of the value. }\end{array}$ \\
\hline
\end{tabular}

In addition, we developed MultiWii control software and drone control trainer board. The drone control trainer board is a board that can simulate the output value and performance of each part by directly mounting each parts of the drone to the PCB board. Through this, the drone was mounted on the fixed PCB board of the trainer, and the drone was mounted so that the simulation could be done. Figure 4 shows the drone pilot trainer board.

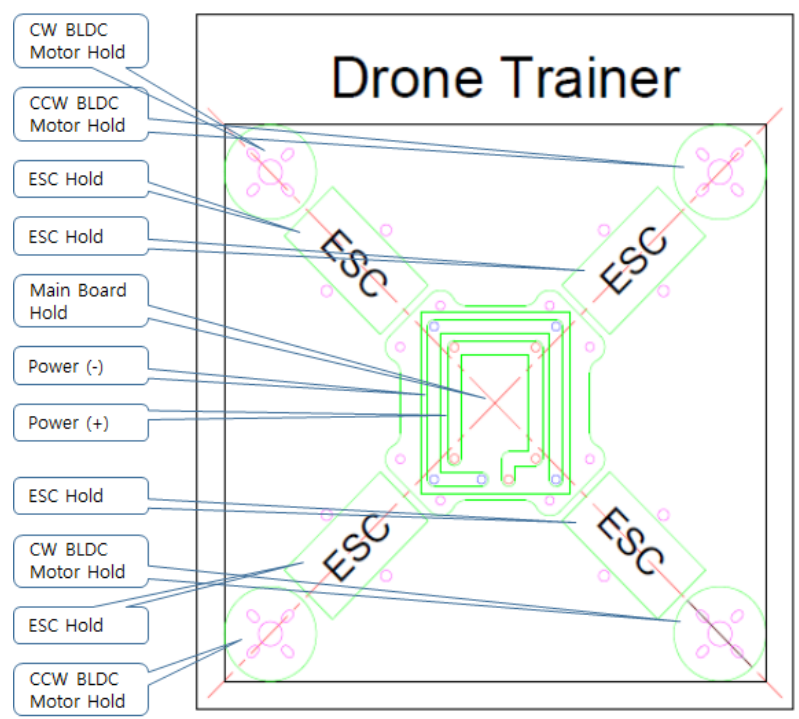

Fig. 4: Drone Pilot Trainer Board Linked with Multiwii.

\section{Conclusion}

The industrial importance of drones and the market size are increasing day by day. Drones' aviation, communication, sensor, and software technologies have a significant impact on related fields. It is also an optimal test bed that can apply and verify the common core technology of the fourth industrial revolution such as artificial intelligence and objects internet and sensor. Also, Drones are required to have positioning technology using acceleration technology, sensor technology such as geomagnetism and pressure, navigation technology to navigate to the target point, and control technology to keep the airframe stable even when the wind is blowing. As such, as the interest in drones increases, the field of utilizing them is also steadily increasing. The drone market is 
expected to improve greatly in the future, and the utilization rate will also increase.

But there are also a few side effects. There are many issues to consider in order to use drones more safely and universally, such as safety and privacy. Until now, the use of drones and the market are in the early stages of development, so it is necessary to have a somewhat flexible regulatory operation in order to develop related industries. However, it is predicted that not only the related technology development and private use will proceed at a rapid pace in the future, but the influences to be brought about in everyday life will be very great. In the end, it is essential to develop and introduce a system that makes the practice tests necessary for drone manipulation and operation safer and through which it is qualified.

Therefore, in this paper, we implemented a central control system for safer drone education and maneuvers. In addition, we have developed software that enables safe pilot test in conjunction with existing fixed drone operating platform. Finally, the control method of the drone can be fully learned and learned in advance through the central control system. In addition, it is possible to acquire application skills of effective drone by measuring the lift and thrust generated while controlling the drone.

\section{References}

[1] Henry, H. Perritt, Jr. Eliot, \&O. Sprague. (2015). Drones. VAND. J. ENT. \& TECH. L., 17(3), 5-15.

[2] Melekhova, A.\&Vinicio, V. (2015). Cloud and grid. Part I: Difference and convergence. Indian Journal of Science and Technology, 8(29), 1-10.

[3] Eresmaa, N. Karppinen, A. M.Joffre, S. Rasanen, J. \&Talvitie, H. (2006). Mixing height determinationby ceilometer.Atmospheric Chemistry and Physics, 6, 1485-1493.

[4] Kim, B. H. (2016). Development of Young Children Coding Drone using Block Game. Indian Journal of Science \& Technology, 9(44), 1-4.

[5] Bart C. (2016). The Future of Drone Use: Opportunities and Threats from Ethical and Legal Perspectives. Asser press.

[6] Sajal, S. Ankit, M. Rajesh, S. \& Anita, G. (2016).UAV for Surveillance and Environmental Monitoring.Indian Journal of Science and Technology, 9 (43), 1-3.

[7] Katsu Y. (2003). Controlling a Marionette with Human Motion Capture Data. Proceedings of the 2003 IEEE International Conference on Robotics and Automation, 3834-3841.

[8] Banu, Y. Zumrut, A.Hakan, B. \& Cigdem, S. (2011). The Effects of Apilarnil (Drone Bee Larvae) Administration on Growth Performance and Secondary Sex Characteristics of Male Broilers. Journal of Animal and Veterinary Advances, 10 (17), 2263-2266.

[9] Altug, E. Ostrowski, J. P. \& Taylor, C. J. (2005). Quadrotor Control Using Dual Camera Visual Feedback.The International Journal of Robotics Research, 24 (5), 329-341.

[10] Abdelhamid, T. \& Stephen, M. (2006). Attitude Stabilization of a VTOL Quadrotor Aircraft. IEEE Trans. On Control System Technology, 14 (3).

[11] Oke, T. R. (1982). The energetic basis of the urban heat island, Quarterly journal of the royal meteorological society, 108(455), 124.

[12] Brouwer. (2015). Surfzone Monitoring Using Rotary Wing Unmanned Aerial Vehicles. Journal of atmospheric and oceanic technology, 32(4), 855-863.

[13] Sørensen, L. Y. Lars Toft Jacobsen, \& John Paulin Hansen. (2017). Low Cost and Flexible UAV Deployment of Sensors. MDPI Sensors, 17 (1), 154. 